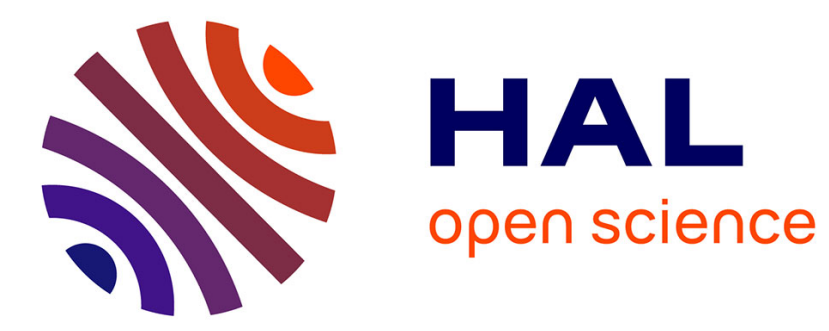

\title{
La conservation du patrimoine khmer aujourd'hui
}

Franciscus Verellen

\section{To cite this version:}

Franciscus Verellen. La conservation du patrimoine khmer aujourd'hui. Comptes-rendus des séances de l'Académie des inscriptions et belles-lettres, 2009, 2010.1, pp.461-474. halshs-02427054

\section{HAL Id: halshs-02427054 \\ https://shs.hal.science/halshs-02427054}

Submitted on 24 Feb 2022

HAL is a multi-disciplinary open access archive for the deposit and dissemination of scientific research documents, whether they are published or not. The documents may come from teaching and research institutions in France or abroad, or from public or private research centers.
L'archive ouverte pluridisciplinaire HAL, est destinée au dépôt et à la diffusion de documents scientifiques de niveau recherche, publiés ou non, émanant des établissements d'enseignement et de recherche français ou étrangers, des laboratoires publics ou privés. 


\section{La conservation du patrimoine khmer aujourd'hui}

\section{Monsieur Franciscus Verellen}

\section{Citer ce document / Cite this document :}

Verellen Franciscus. La conservation du patrimoine khmer aujourd'hui. In: Comptes rendus des séances de l'Académie des Inscriptions et Belles-Lettres, 154e année, N. 1, 2010. pp. 461-474;

doi : https://doi.org/10.3406/crai.2010.92830

https://www.persee.fr/doc/crai_0065-0536_2010_num_154_1_92830

Fichier pdf généré le 19/07/2018 


\title{
LA CONSERVATION \\ DU PATRIMOINE KHMER AUJOURD’HUI
}

\author{
PAR M. FRANCISCUS VERELLEN, \\ MEMBRE DE L'ACADÉMIE
}

Sire,

C'est une grande joie, pour le directeur de l'École française d'Extrême-Orient, d'être aux côtés de ses confrères académiciens, pour la cérémonie qui installe Votre Majesté à l'Académie des Inscriptions et Belles-Lettres.

Lors de Sa visite d'État en France en 2006, je me rappelle que Votre Majesté a fait grand honneur à l'EFEO en rendant hommage [je cite] « aux éminents savants qui bâtirent son prestige », et en saluant dans les chercheurs de l'École aujourd'hui « les dignes successeurs de ceux-ci ». Comme notre Président Pierre-Sylvain Filliozat l'a évoqué, voilà plus d'un siècle que l'École française d'ExtrêmeOrient s'attache à l'étude du Cambodge (fig. 1), à la recherche de son histoire, de son génie, de ses inscriptions et belles-lettres au sens où notre Secrétaire perpétuel aime à définir ces termes.

Il m'échoit cet après-midi d'évoquer les travaux de ses chercheurs, et, en prélude, permettez moi de Vous exprimer toute ma reconnaissance pour l'accueil et le soutien dont bénéficie grâce à Vous notre établissement, tant auprès du Ministère de la Culture du Royaume du Cambodge, de l'Autorité pour la Protection du Site et l'Aménagement de la Région d'Angkor APSARA, que de l'UNESCO où Vous avez considérablement œuvré comme ambassadeur pour la préservation et la sauvegarde d'Angkor, aux côtés de Sa Majesté le roi-Père Norodom Sihanouk.

Certes, les mots ne sont que de pâles miroirs devant les splendeurs de la civilisation d'Angkor que les scientifiques de l'École ont le privilège de révéler chaque fois un peu plus au grand jour. Je ressens ainsi beaucoup d'humilité à évoquer devant Vous la complexité des grands travaux de terrain auxquels les équipes de l'EFEO se livrent sans relâche, à commencer par celle dont le chantier mis en œuvre 


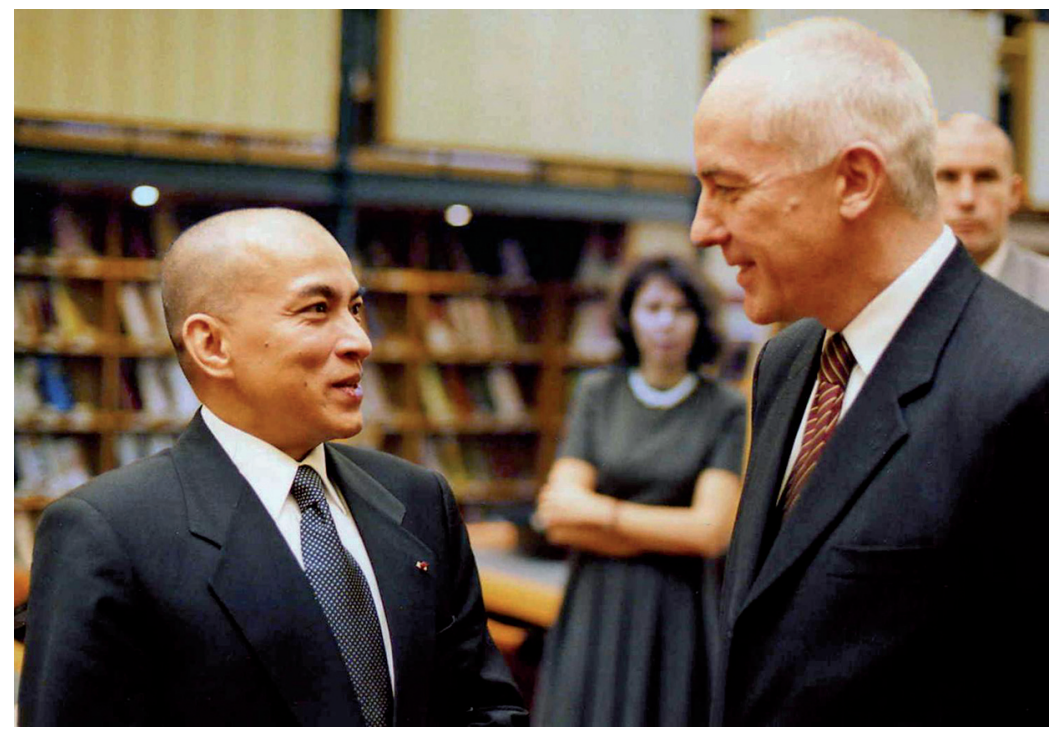

FIG. 1. - Visite de Sa Majesté le roi Norodom Sihamoni à l'EFEO, à l'occasion de sa visite d'État en France en 2006 (photographie : Sara Darmayan).

à Angkor s'est fixé pour tâche d'étudier les «Cités anciennes et la Structuration de 1'espace en Asie du Sud-Est».

Chers confrères, c'est sous un angle de recherche comme celuilà que vous comprendrez le mieux, je n'en doute pas, la fierté que j'éprouve à animer les travaux de l'École française d'Extrême-Orient.

Nous voici donc au cœur de la « Grande Ville» royale d'Angkor Thom, devant le gigantesque dépôt lapidaire du Baphuon (fig. 2), structure montagne de 100 mètres par 150 mètres au sol - 30 mètres de hauteur, dont les travaux de restauration furent repris le 21 février 1995, au cours d'une cérémonie solennelle présidée par Sa Majesté le Roi-Père. Il s'agit aujourd'hui d'un chantier herculéen de 240 personnes, dirigé par notre collègue Pascal Royère - lauréat en 2006 du Grand Prix de la Fondation Prince Louis de Polignac.

Véritable puzzle en trois dimensions, il a fallu fouiller la terre, dégager d'énormes éboulis, retrouver le tracé original des murs, identifier patiemment trois cent mille blocs de grès (fig. 3) sur plus de 10 hectares, redéfinir les plateformes, reconstruire les gopura et les galeries des étages supérieurs. Dans quelques mois, les dernières pierres retrouveront leur place sur le sommet sacré, et la haute silhouette de la tour centrale du Baphuon, effondrée depuis des siècles, sera restituée aux regards des hommes. 


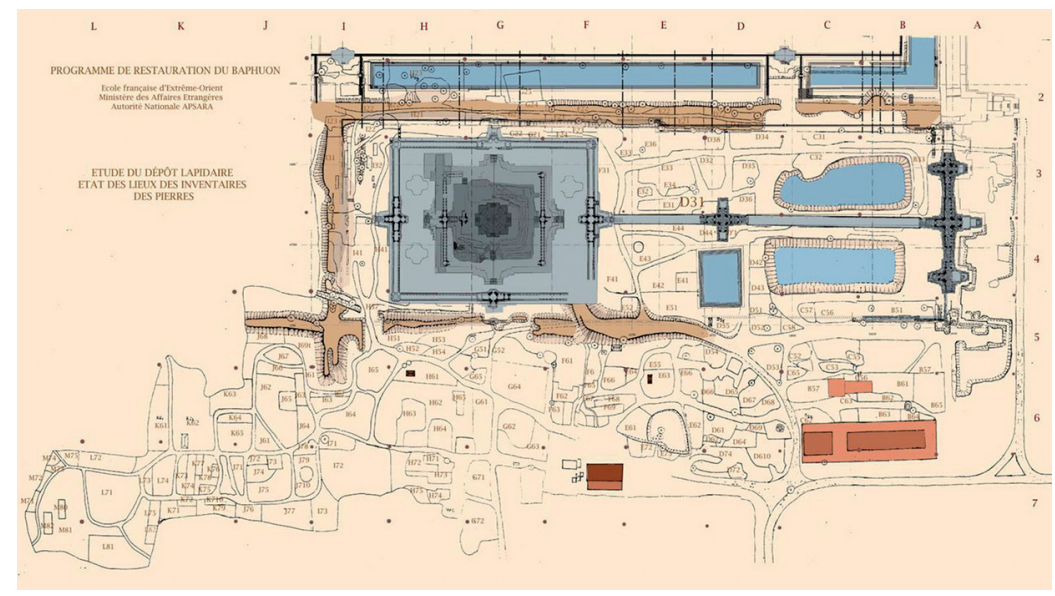

FIG. 2. - Temple montagne du Baphuon, plan et schéma du dépôt lapidaire (photographie : Pascal Royère).

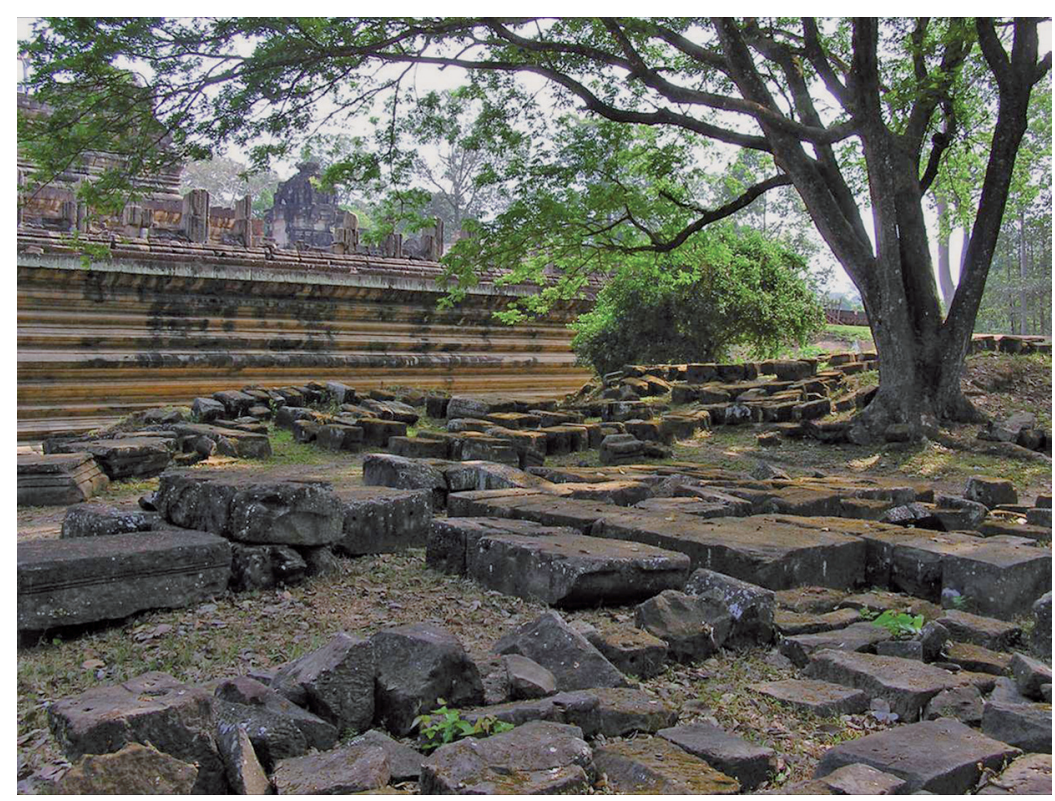

FIG. 3. - Temple montagne du Baphuon, dépôt lapidaire devant le soubassement du temple (photographie : Pascal Royère). 
Mais ce n'est pas tout. La reconstruction du Baphuon a mieux fait comprendre les idées qui animaient l'esprit des bâtisseurs d'Angkor. Les sanctuaires étaient aussi les pavillons de passage où sont nées les premières intentions de bas-reliefs, dont le développement aboutira aux grands pans de mur sculptés des temples plus tardifs d'Angkor Vat (fig. 4) et du Bayon. Son approche scientifique, «de l'intérieur », a permis de collecter toute une documentation sur l'histoire des techniques, les processus de construction, et d'introduire dans notre réflexion de nouvelles notions sur les séquences temporelles, sur les phases de réaffectation et de refonte religieuse du temple.

De ce point de vue, dans cet ensemble du $\mathrm{XI}^{\mathrm{e}}$ siècle originellement sivaïte, le grand Bouddha couché qui occupe depuis la seconde moitié du XVI $\mathrm{XI}^{\mathrm{e}}$ tout le côté ouest du deuxième étage, devient le spectaculaire témoignage des transformations qui ont fait bouger l'histoire du Cambodge. La restauration de cette statue gigantesque de 73 mètres de long fut marquée par une cérémonie de consécration, que Votre Majesté nous a fait 1'honneur de présider le 4 juin 2008 (fig. 5). Vous étiez d'ailleurs déjà venu dix ans plus tôt, au moment des travaux de démontage préventif, et une deuxième fois en 2000, à l'occasion des célébrations du centenaire de l'EFEO, sous la direction de mon estimé prédécesseur Jean-Pierre Drège.

Chers confrères,

je vous invite maintenant à vous transporter loin du cliquetis des burins (fig. 6), du bruit des grues, de la rotation des bennes, du grincement des paniers en bambou et des échafaudages, pour retrouver le silence au milieu des grands arbres d'Angkor Thom. C'est à la demande du regretté directeur de l'EFEO Denys Lombard, que notre collègue archéologue Jacques Gaucher a entamé en 2000 un nouveau programme de recherche intitulé «De Yasodharapura à Angkor Thom, ville et archéologie », fruit d'une collaboration incluant l'EFEO et l'APSARA, dans le cadre budgétaire du Fond de Solidarité Prioritaire «Angkor : site, patrimoine et développement » du Ministère des Affaires étrangères et européennes.

Il s'agit là du premier programme de recherche archéologique systématique à l'échelle globale, au niveau du foyer de l'empire angkorien, c'est-à-dire non pas tant du point de vue des temples que de la cité elle-même. Ici, les obstacles physiques ne manquent pas : couverture végétale à $80 \%$, vastes étendues au milieu d'une enceinte carrée de trois kilomètres de côté, sédimentation, mines. Sa problématique archéologique est des plus complexes : mettre à 


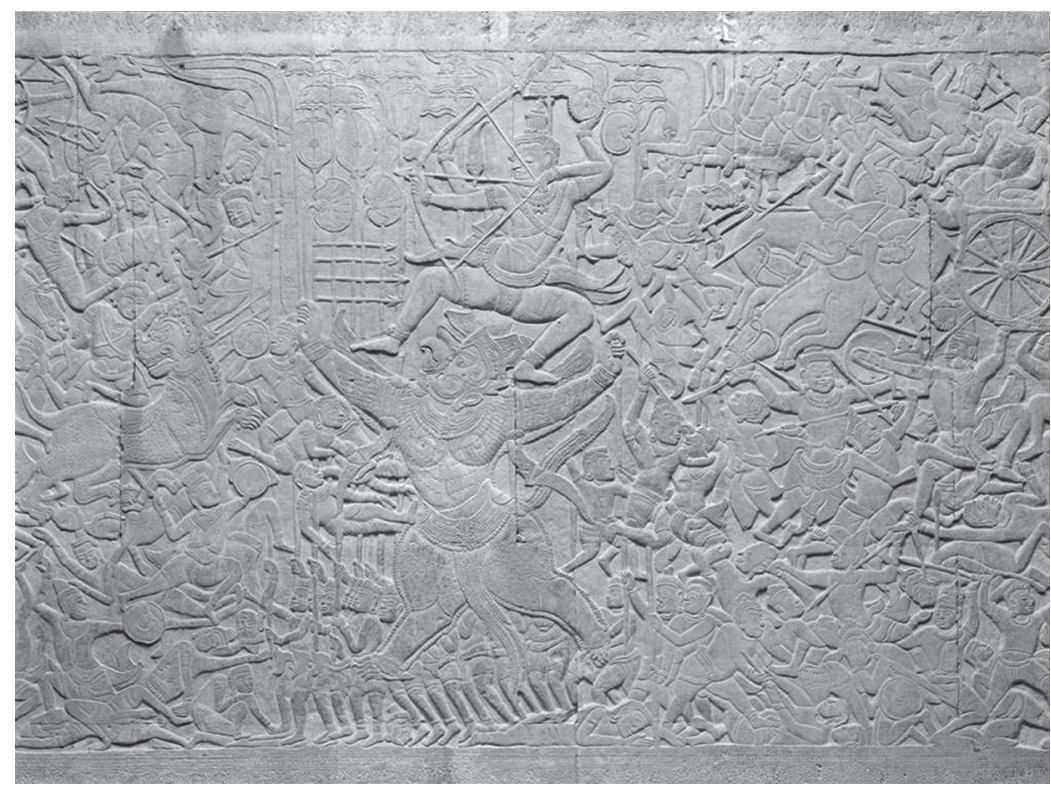

FIG. 4. - Bas-relief des grandes galeries d'Angkor Vat, le dieu Vishnou sur sa monture l'aigle Garuda (photographie : Luc Ionesco).

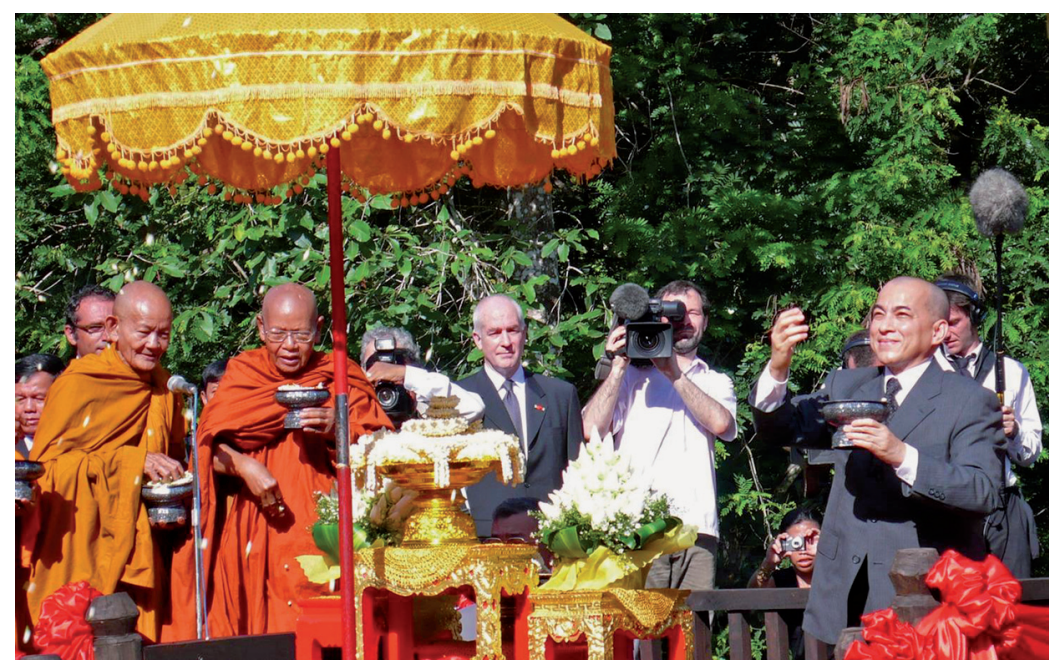

FIG. 5. - Consécration du Bouddha couché de la façade ouest du temple du Baphuon par Sa Majesté le roi Norodom Sihamoni en présence du directeur de l'EFEO, Franciscus Verellen, juin 2008 (photographie : Philippe Bataillard). 


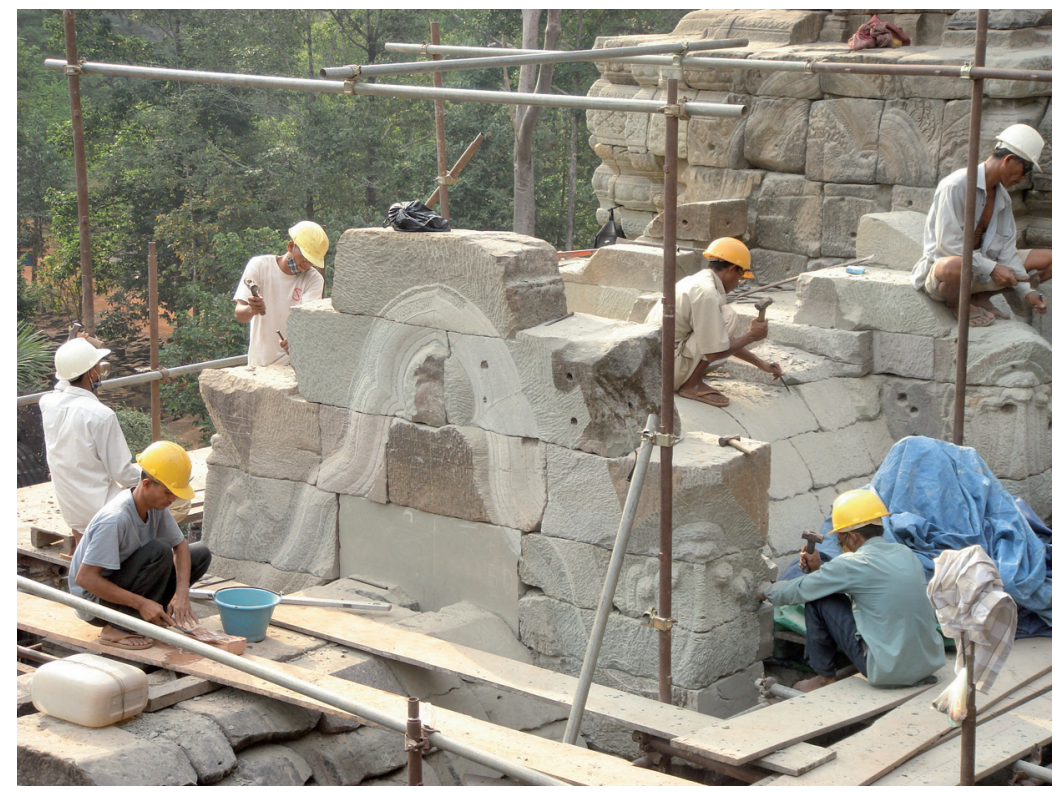

FIG. 6. - Chantier du temple montagne du Baphuon, tailleurs de pierre (photographie : Pascal Royère).

jour l'organisation urbaine d'un espace de 900 hectares, en saisir la fabrication à travers ses temporalités et ses fonctionnements, rendre compte des ruptures et des permanences sur les plans spatiaux, politiques et sociaux.

Durant la première phase du projet, l'enregistrement, l'analyse et le croisement des informations obtenues du sol archéologique et de la surface du sol, a permis de cartographier l'ancienne ville (fig. 7), horizontalement et verticalement, et d'affirmer tout de suite que la figure centrale du site d'Angkor recouvrait celle d'une capitale. À un moment de son histoire, celle-ci avait donc été densément habitée. Il ne s'agissait pas d'une ville neuve : c'était le fruit d'une fabrication urbaine étalée sur de nombreux siècles.

La seconde phase vise la chronologie des grandes évolutions du site et l'impact que l'introduction soudaine du bouddhisme cinghalais a laissé. Un cadastre fossile, à l'échelle du $1 / 2000^{\circ}$, permet d'ores et déjà d'appréhender la topographie sociale d'Angkor Thom, ses modes de distribution de l'habitat, son évolution en îlots urbains : plus de $150 \mathrm{~km}$ de rues et de voies d'eau découverts, près 


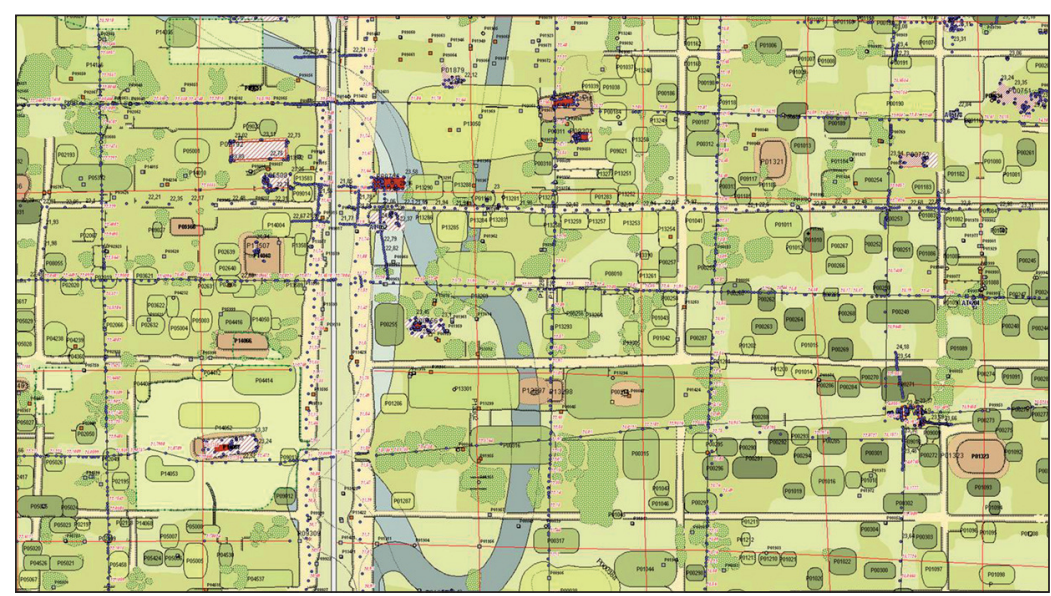

FIG. 7. - Topographie urbaine d'Angkor Thom (extrait du nouveau plan de la ville archéologique) : voies d'eau, habitat, bassins, vestiges (Jacques Gaucher).

de 350 îlots d'habitation recensés, plus de 3000 bassins dénombrés, plus de 200 vestiges repérés.

Les fouilles du dispositif monumental des enceintes ont mis en évidence le fait que la Grande douve (fig. 8) et les murs d'Angkor Thom (fig. 9) ne sont pas des créations ex nihilo mais reposent sur un tracé encore plus ancien. De même, les fouilles sur la zone du Phimeanakas (fig. 10) ont montré que les premières occupations du site sont antérieures, elles aussi, à la construction du Palais royal.

Bref, 1'étude d'Angkor Thom sous cet angle nouveau est une découverte colossale. La cité entière est un monument dont la restauration relève de l'archéologie du paysage. En 2008, le prix Ikuo Hirayama a été décerné par notre Académie à Jacques Gaucher pour sa publication du plan archéologique de la ville.

Sire,

avant de quitter Angkor Thom, permettez moi d'évoquer aussi le programme archéologique "Angkor Medieval Hospitals », que mène sur place notre collègue Christophe Pottier, et que Vous connaissez bien puisqu'il est placé sous Votre haut patronage. Ce programme s'intéresse à l'un des quatre temples-hôpitaux dédié au Bouddha médecin, et construits au XII ${ }^{\mathrm{e}}$ siècle par Jayavarman VII.

Le travail de la Mission Archéologique Franco-Khmère sur l'Aménagement du Territoire Angkorien (MAFKATA), nous fait embrasser, en prenant cette fois de l'altitude, les 40000 hectares 


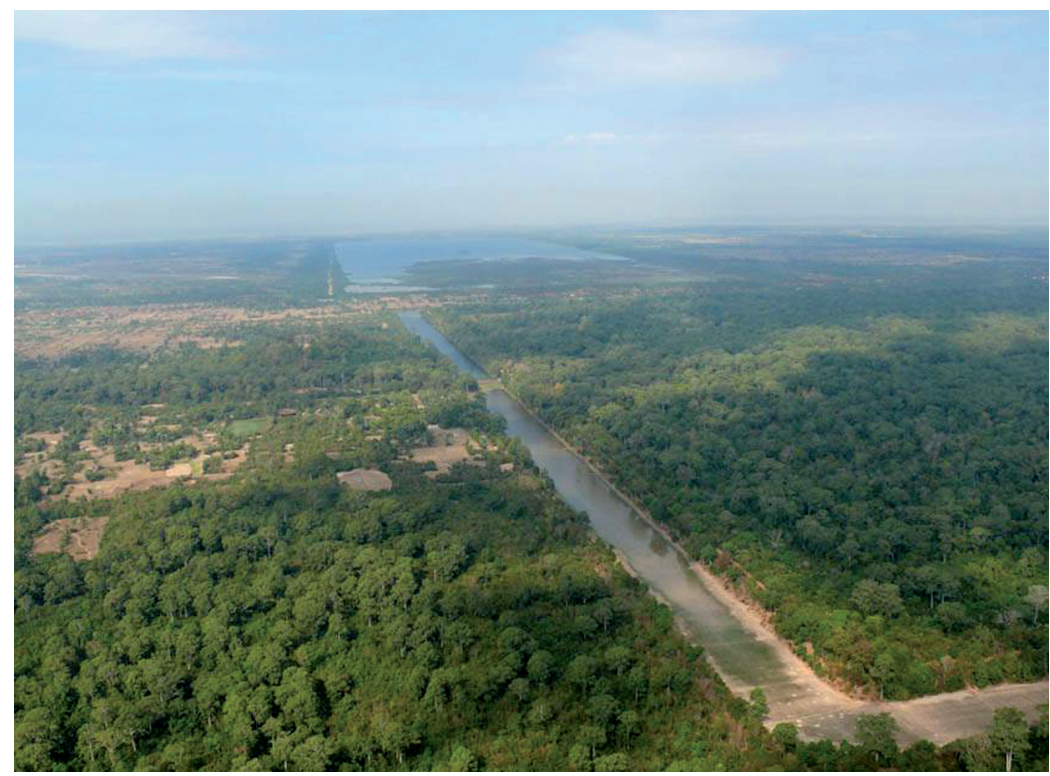

FIG. 8. - Les douves d'Angkor Thom, au second plan le baray occidental (photographie : Jacques Gaucher).

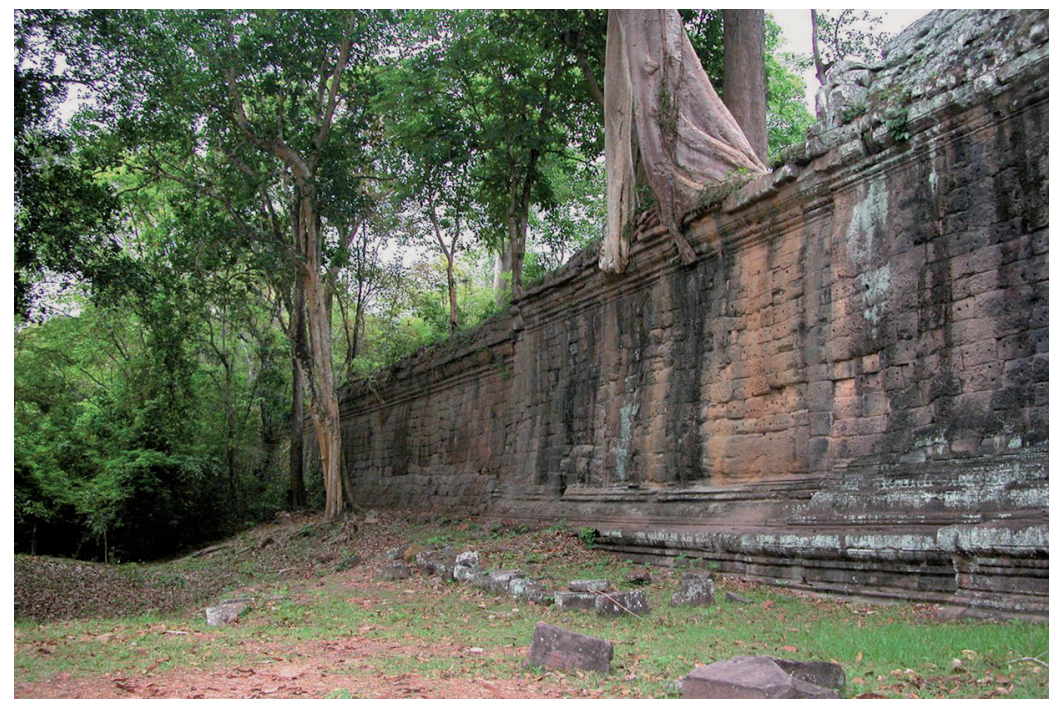

FIG. 9. - Angkor Thom, murs d'enceinte de la ville (photographie : Jacques Gaucher). 


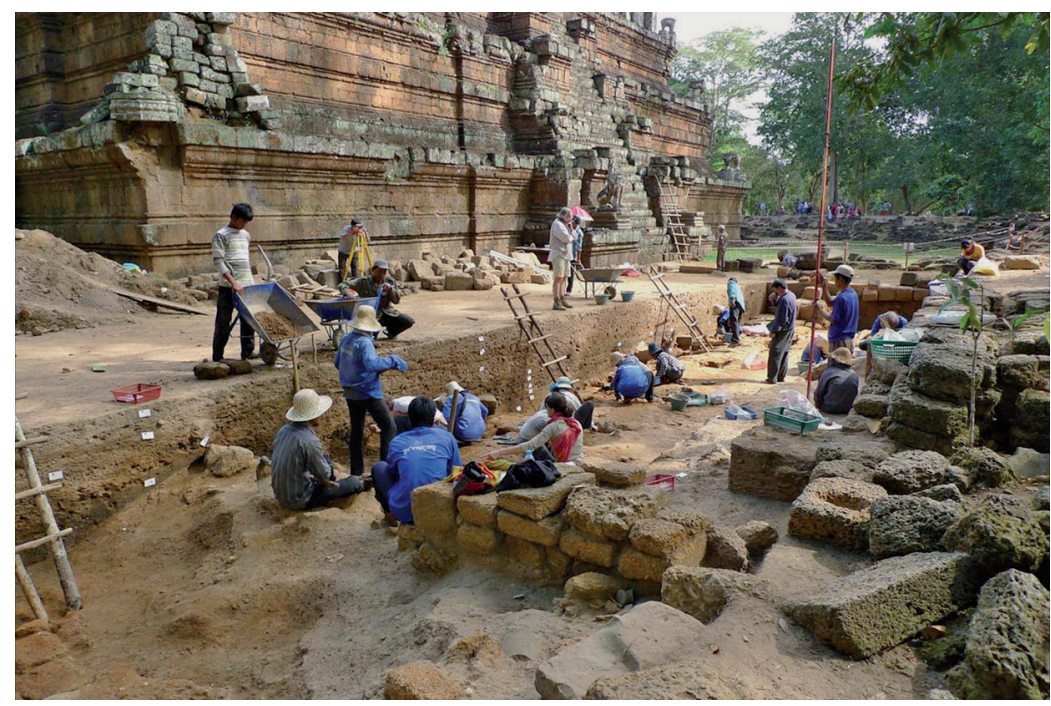

FIG. 10. - Angkor Thom, fouilles devant le temple du Phimeanakas (photographie : Jacques Gaucher).

du parc d'Angkor, avec ses villages centenaires qui donnent vie au paysage, ses petites plantations, ses rizières, ses nombreuses espèces de palmiers, ses « fromagers », ses Dipterocarpus et autres gommiers de 50 mètres. Ici, la recherche utilise tous les moyens d'investigation - repérage aérien, télédétection, prospection Géoradar, susceptibilité et résistivité magnétique - pour l'étude morphologique, environnementale et historique de l'occupation du territoire d'Angkor. Mené par Christophe Pottier en association avec l'Université de Sydney, ce programme de l'EFEO a reçu l'approbation du Comité international de coordination pour Angkor en 2000, ainsi que le soutien du Ministère des Affaires étrangères et européennes. Plusieurs centaines de nouveaux sites et d'installations préhistoriques ont été mis à jour, obligeant à reconsidérer la densité des occupations, les modalités d'aménagements, la genèse de l'urbanisme, jusqu'au concept de territoire dans les « villes » angkoriennes. Ces travaux de grande envergure ont été encouragés par le prix Carroll de notre Académie en 2008.

Dans la foulée, l'exposition «Les ancêtres d'Angkor», organisée en 2009 par le musée national de Phnom Penh, l'EFEO et l'Autorité nationale APSARA, avec le soutien de l'UNESCO, a présenté une sélection de données et d'artefacts préhistoriques 


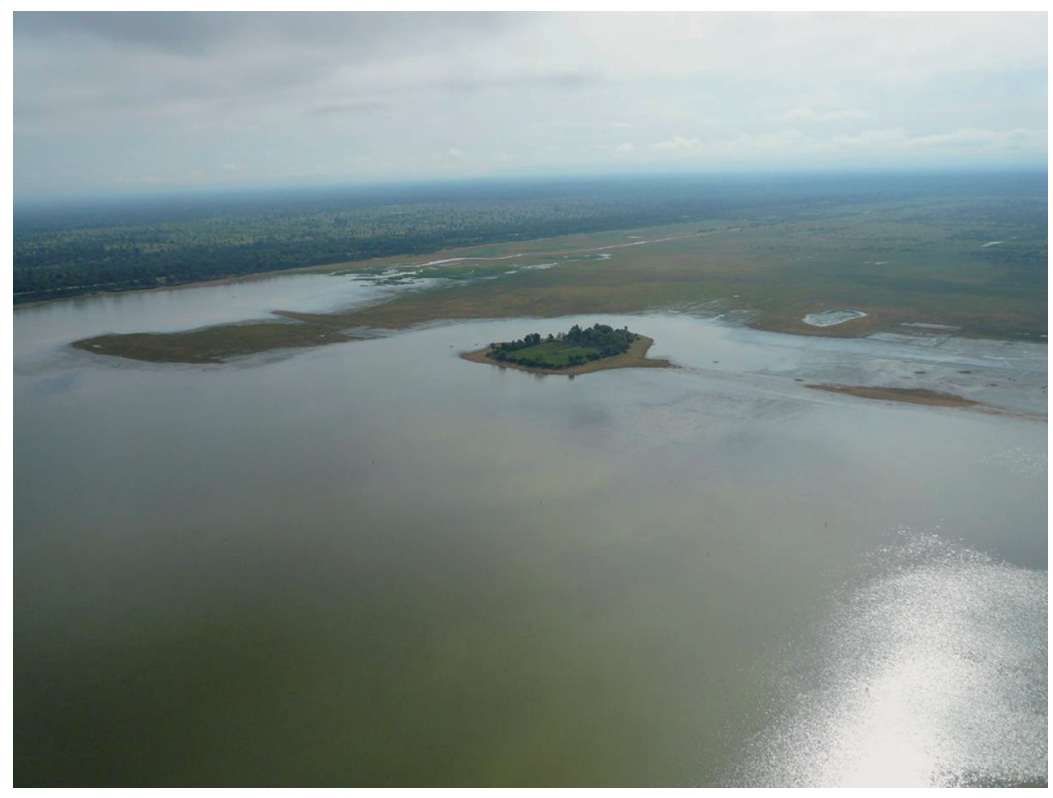

FIG. 11. - Baray occidental. Temple du Mebon occidental ; sous les eaux site de Koh Ta Meas, vestige d'une nécropole de l'âge du Bronze (photographie : Christophe Pottier).

inédits, qui éclairent les périodes les plus mal connues de l'histoire du Cambodge, en particulier la continuité du site angkorien de Prei Khmeng, du début de l'ère chrétienne jusqu'à l'installation des premiers temples brahmaniques. Quant au site de Koh Ta Meas, situé sous les eaux du baray occidental d'Angkor (fig. 11), il révèle les vestiges d'une nécropole datant de l'âge du Bronze de plus de 3500 ans.

C'est à l'ensemble de tous ces sites archéologiques du Cambodge que s'intéresse depuis 1990 notre collègue de l'EFEO Bruno Bruguier. Les prospections de terrain de grande envergure menées en partenariat avec le Ministère de la Culture du Cambodge et associées, en 2002, au programme «Prévention du trafic illicite des objets d'art » du Ministère des Affaires étrangères et européennes ont nourri une base de données unique : archives, photos, plans et publications de l'EFEO depuis un siècle. Elle intègre désormais des notices sur plus de deux mille sites et trois mille nouveaux points géographiques. 


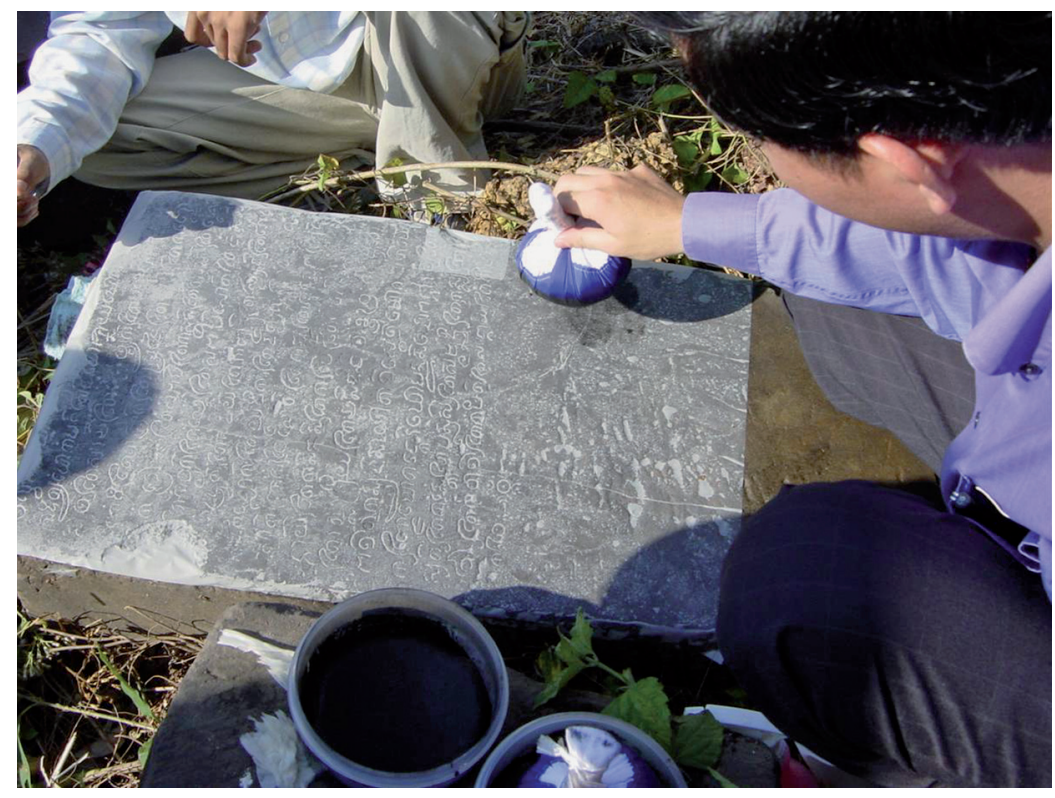

FIG. 12. - Estampage d'une inscription inédite d'une chapelle d'hôpital dans le cadre du projet «Espace khmer ancien » (photographie : Christophe Pottier).

En 2008 est né le projet « Espace Khmer Ancien : Construction d'un corpus numérique de données archéologiques et épigraphiques » (fig. 12), dirigé par notre collègue Pierre-Yves Manguin. Il s'agit d'un système de gestion et de valorisation des vastes collections qui continuent de s'accumuler, tant pour les historiens, les archéologues, les épigraphistes, les architectes, que pour un public plus large. Le projet s'est associé avec le programme joint EPHE-EFEO intitulé « Corpus des Inscriptions khmères », dirigé par notre collègue Gerdi Gerschheimer depuis 2004, sur le modèle des inventaires établis par notre éminent et regretté confrère George Cœdès, repris entre temps avec distinction par notre collègue Claude Jacques.

Sire, Chers confrères,

délaissant un instant l'espace khmer ancien proprement dit (fig. 13), qui déborde d'ailleurs de beaucoup les limites actuelles du Cambodge, je vous invite à traverser le Tonlé Sap pour rejoindre Phnom Penh, juste au nord du Palais Royal, au cœur du Musée national. Nous voilà dans l'atelier de restauration (fig. 14) où notre collègue Bertrand Porte contribue, par la pratique et la formation, à la sauvegarde des œuvres sculptées. La restauration actuellement la plus marquante 


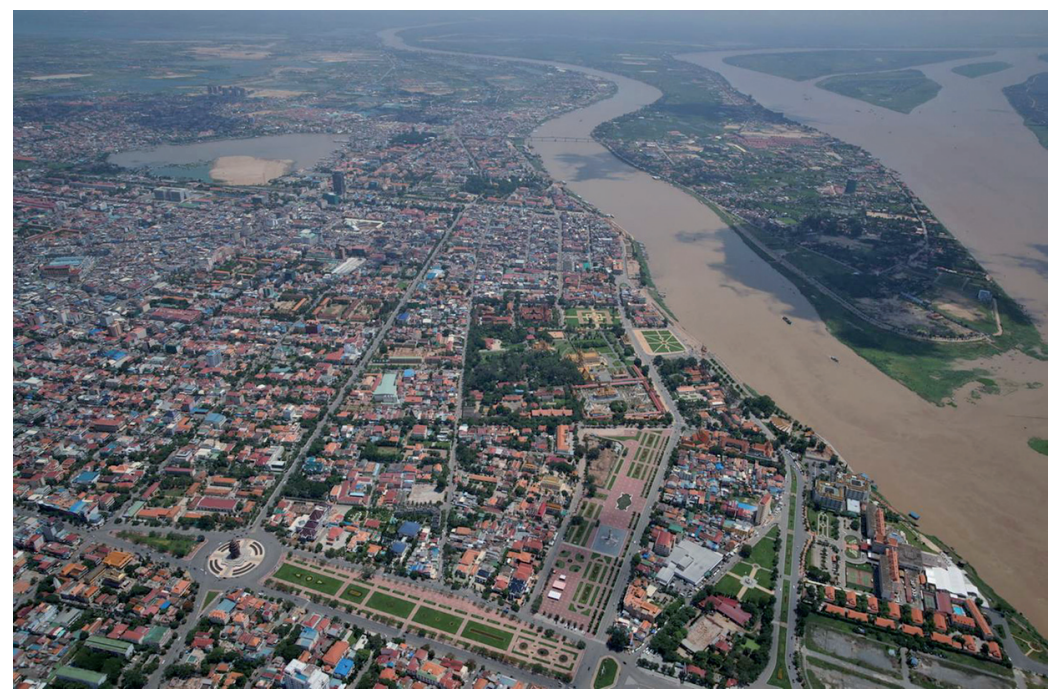

FIG. 13. - Photographie aérienne du Palais Royal de Phnom Penh, capitale du Cambodge (PGEC/ EFEO).

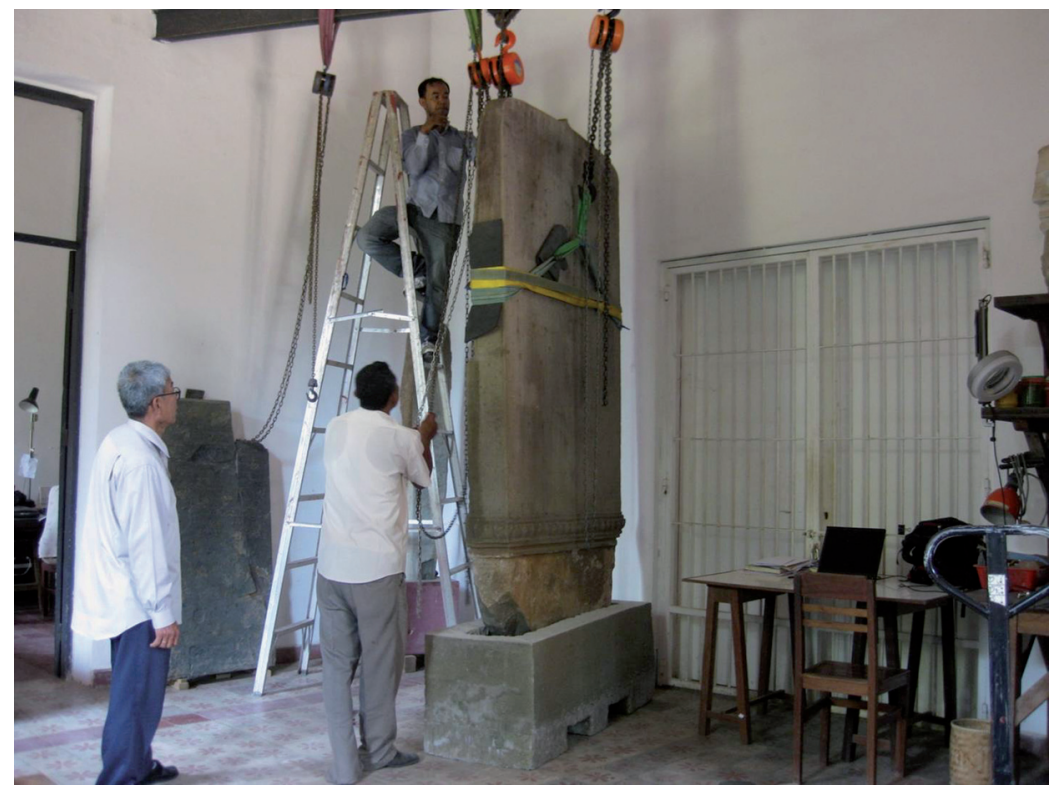

FIG. 14. - Atelier de restauration du musée national de Phnom Penh, installation de la stèle de Pré Rup (photographie : Bertrand Porte). 
concerne la tête et le buste de la statue colossale en bronze du Vishnu narayana, retrouvée au centre du baray occidental d'Angkor.

C'est dans cet esprit d'une mise en valeur des collections archéologiques, que le catalogue intitulé « L'art du Cambodge ancien - les collections du Musée national de Phnom Penh », a été publié par Nadine Dalsheimer en 2001, avec une présentation de notre estimé collègue Bruno Dagens. Ce catalogue établit les notices détaillées d'une sélection de 172 œuvres - statuaire, éléments architecturaux, stèles inscrites, objets en bronze, objets en bois - parmi 4500 pièces inventoriées.

Sire, Chers confrères,

si le sol khmer nous parle, que dire de la mémoire des textes manuscrits du bouddhisme en langue vernaculaire (fig. 15), dont regorgeaient plus que nulle part ailleurs les monastères du Cambodge? C'est pour répondre à cette interrogation cruciale - parce qu'elle touche au cœur du riche patrimoine littéraire et religieux non seulement du Cambodge, mais des autres pays bouddhistes d'Asie du Sud-Est, à la suite des Khmers -, que dans le prolongement de ses travaux sur l'origine des écoles bouddhiques du Cambodge, notre estimé collègue François Bizot a créé en 1989 le «Fonds pour l'édition des manuscrits ». Soutenu par les Affaires étrangères, ce projet EFEO destiné à préserver, à étudier et à traduire les textes du bouddhisme, a pris corps à Phnom Penh sous le nom de FEMC - « Fonds d'édition des manuscrits du Cambodge »-, par un accord de coopération avec le Ministère cambodgien de la Culture signé en 1991. L'antenne cambodgienne, placée sous la protection du haut patronage royal dès 1993 , et conduite sur place par notre collègue Olivier de Bernon, reçut dans un premier temps la mission de sauver les derniers manuscrits rescapés de l'autodafé perpétré par le régime des Khmers rouges.

Chaque texte retrouvé est aujourd'hui traité in situ, sous le regard des moines et avec leur participation, dépoussiéré, identifié, décrit, mesuré, nettoyé feuillet par feuillet, réparé, puis photographié. Les liasses sont soigneusement reconstituées et conservées dans des armoires offertes sur place au monastère. En 19 ans de prospections ininterrompues, cent soixante mille clichés ont été numérisés. Avec le concours de l'UNESCO, une base de données de l'ensemble déjà récolté est en voie d'élaboration. Si les bureaux et le laboratoire du FEMC sont hébergés depuis 1999 au Vat Unnalom, résidence du 


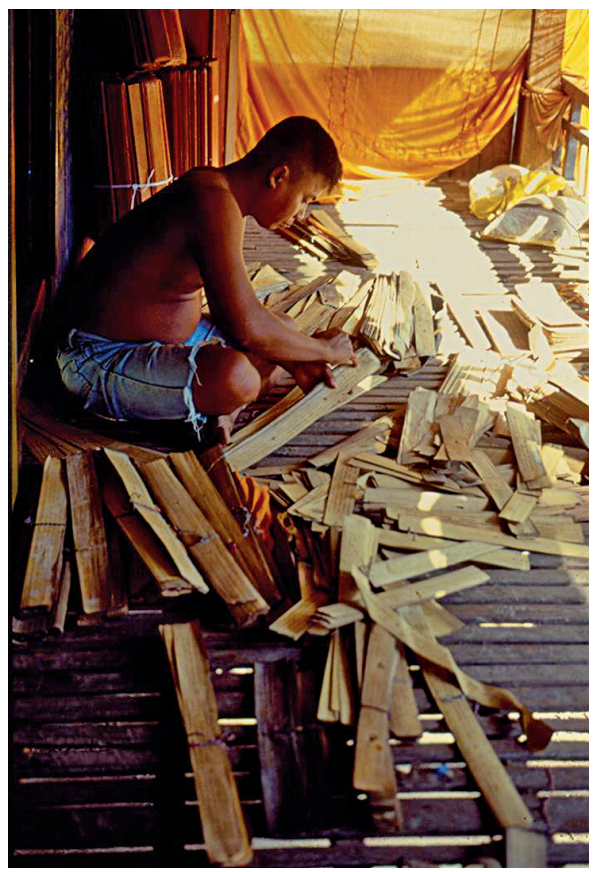

FIG. 15. - Fonds d'édition des manuscrits du Cambodge, reconstitution d'un manuscrit sur ôles (photographie : Olivier de Bernon).

Suprême Patriarche du Cambodge, ils ont longtemps été installés dans l'enceinte même du Palais Royal où Vous résidez.

Sire,

au terme de ce retour éclair sur les sources actuelles de la mémoire des Khmers, notre Compagnie n'aura pu être que saisie par le rôle primordial de Votre Majesté en faveur de la recherche historique, archéologique et philologique. Le soutien décisif que Votre Majesté apporte aux travaux de l'École française d'Extrême-Orient est un encouragement pour tous. Cela appelle chacun d'entre nous à se porter encore plus activement à l'étude et à la sauvegarde du patrimoine cambodgien. Sans attendre, il faut favoriser la formation des nouvelles générations de scientifiques et de spécialistes pour demain.

Puisse l'EFEO et la recherche française, sous Votre haute bienveillance, avec le souci du développement durable dans l'aménagement du territoire, continuer de participer longtemps à l'enrichissement culturel et économique du Royaume du Cambodge, comme à la préservation de ses repères identitaires. 